\title{
Development of Infection Control Surveillance System for Intensive Care Unit: Data Requirements and Guidelines
}

\author{
Manal Abumelha ${ }^{1,2}$, Awatef Hashbal ${ }^{1,2}$, Farrukh Nade em ${ }^{1}$, Naif Aljohani ${ }^{1}$ \\ ${ }^{1}$ King Abdulaziz University, Department of Information Systems, Jeddah, Kingdom of Saudi Arabia \\ ${ }^{2}$ King Khalid University, Department of Information Systems, Abha, Kingdom of Saudi Arabia \\ E-mail: mabomlah@kku.edu.sa, awatef@kku.edu.sa, fabdullatif@kau.edu.sa,nraljohani@kau.edu.sa
}

\begin{abstract}
Surveillance systems are useful in the identification of patients that contract infections during their hospitalization period. Despite still being at infancy, electronic information control surveillance systems for Hospital Acquired Infections (HAIs) are improving and becoming more commonplace as the acceptance levels rise. There are crucial gaps in existing knowledge concerning the best ways for implementing electronic surveillance systems especially in the context of the Intensive Care Unit (ICU). To bridge this gap, the aim of this paper was to provide a comprehensive review of various electronic surveillance approaches and to highlight the requisite data components and offer guidelines. This review revealed denominator, nu merator, and discrete data requirements and guidelines for the surveillance of four main ICU HAIs, including Central Line-Associated Bloodstream Infection (CLABSI), Urinary Tract Infection (UTI), Surgical Site Infections (SSIs) and Ventilator-Associated Conditions/Events (VACs/VAEs).
\end{abstract}

Index Terms-Surveillance, Hospital Acquired Infections (HAIs), Central Line-Associated Bloodstream Infection (CLABSI), Urinary Tract Infection (UTI), Surgical Site Infections (SSIs) and Ventilator-Associated Conditions/Events (VACs/VAEs).

\section{INTRODUCTION}

Healthcare-Associated Infections' (HAI) surveillance is one of the important pillars in prevention of infections. This is because HAIs affect the disease burden considerably. To ensure substantive reduction of HAIs, hospitals need to track the type, location, and number of infections that occur within the facility and come up with effective efforts towards target prevention [1]. Surveillance systems are useful in the identification of patients that contract infections during their hospitalization period. They are also useful in identifying patients who enter the hospital with already preexistent infections. This identification assists healthcare staffs in designing and initiating appropriate precautions for limiting infections' spread [1].

In order to ensure that such surveillance remains useful, there is need to adhere to standard definitions that are applicable consistently over a long period and between locations. In addition, such definitions must reflect conditions that are preventable through efforts towards improvement [2]. It is imperative to understand that surveillance definitions differ from clinical diagnostic standards. This is because the purpose of clinical definitions is prognosis and treatment while surveillance definitions are for benchmarking and trending [3]. Conventional surveillance systems tend to be cumbersome, inaccurate and susceptible to high interobserver inconsistency [4]. However, the advent of Electronic Health Records (EHRs) increased the popularity of electronic assistance in the prevention of infections, particularly in case finding [5].

\section{A. Background and History}

Nelson and Safikis [6] defined infectious disease surveillance as "the continuous systematic collection of data on illness or infections in a defined population to monitor the incidence or prevalence of a disease or a behavior that is place people at risk of disease or ill health" (p.117). The origins of infection control surveillance systems are traceable to the beginning of public health surveillance. Public health surveillance dates as far back as $3180 \mathrm{BC}$ following the first ever recorded epidemic in Egypt during the reign of Pharaoh Mempses [7] as cited in [8]. However, Hippocrates came up with the terms epidemic and endemic between $460 \mathrm{BC}$ and $370 \mathrm{BC}$ [9].

The idea of systematic ongoing mortality data collection first emerged in 1532 when the London town council began keeping records of individuals dying from the Bubonic plague [10]. In the 1600s, John Graunt introduced comprehensive analysis and interpretation to become the first person quantifying disease patterns and understanding that numerical data about a population could be useful in studying disease causes [8]. Altogether, William Farr from Great Britain, was the first person to develop the modern surveillance concepts while serving as the superintendent at the Statistical Department of the Registrar General's office of England and Wales between 1839 and 1879 [6]. John Snow pioneered the linkage of data to intervention between 1813 and 1858 whereas Alexander Langmuir was the first person to comprehensively define surveillance between 1910 and 
1993.

\section{B. Aim and Objectives}

Prior to the commencement of electronic surveillance for HAIs, several important considerations relating to the kind of data required in EHRs and the structuring of such data is necessary [2]. There are crucial gaps in existing knowledge concerning the best ways for implementing electronic surveillance systems especially in the context of the Intensive Care Un it (ICU). To bridge this gap, the aim of this paper is to provide a comprehensive review of various electronic surveillance approaches and to highlight the requisite data components and offer guidelines. Subsequently, this review addressed the following objectives:

1. To review the different electronic surveillance approaches in the context of ICUs

2. To analyze the data requirements of Infection Control Surveillance System in ICUs

3. To offer guidelines concerning data requirements of Infection Control Surveillance System in ICUs

The next two sections contain the review of data requirements starting with the general data requirements. It also includes the data requirements for the main ICUrelated HAIs. The recommendations for the development of information control surveillance systems related to the data requirements reviewed also appear in the last section.

\section{DAT A REQUIREMENTS}

There are some data requirements in order to control the infection by using surveillance system in the intensive care unit.

\section{A. General Data Requirements}

Majority of HAI criteria for undertaking surveillance such as those issued by the Centers for Disease Control and Prevention/National Healthcare Safety Network (CDC/NHSN) entail sympto ms and signs including fever, details of medical procedures such as surgery duration, and data pertaining to medical device exposure like ventilators as well as microbiological and laboratory test results [2]. Electronic surveillance systems often rely on data timeliness, accuracy and consistency on the said exposures and signs. Strategies geared towards enhancing data collection quality include minimization of data entry involving free text in addition to reorganization of documentation to significantly structured, distinct patches. Additionally, cultivation of institutional cultures that welcome technology and ones with positive views towards change could enhance data quality [11]. In the next three subsections the authors focuses on data capturing with distinctive review of denominator, numerator and discrete data capturing respectively.

\section{1) Denominator Data Capturing:}

Valid enumeration of procedures, days, and at-risk patients are essential elements to capturing accurate rates of infection. This process features underappreciated complexities. For instance, denominator data in Surgical Site Infections (SSIs) come from the procedures' count. Correct evaluation of the data could prove problematic since competing systems of coding, for example, Current Procedural Terminology (CPT) and International Classification of Diseases Ninth Revision (ICD-9) that do not have a 1:1 mapping. Infections associated with devices for which the expression of denominators is in relation to device days is another example. In the case of Central Line-Associated Bloodstream Infection (CLA BSI), cu rrent standards involve tally ing one deviceday if a patient presents with Central Venous Catheter (CVC), the number of present lines notwithstanding [12].

Manual surveillance involves tallying each day at the same time [4]. Electronic surveillance has the capacity to give different results, particularly when tallying devicedays is based on proof of availability of whatever line within a timeframe of 24 hours. In order to ensure error reduction, documentation within an EHR requires construction to eliminate ambiguity [13]. For instance, the actual anatomic site and device need capturing as discrete data. The involvement of infection preventionists while designing automated documentation is important for enumerating the device-days successfully, consistently, and accurately [2]. When programmers have records with sufficient detailing, they get the potential to reduce inconsistencies between manual and electronic device counts.

Additionally, garnering detailed data through all devices would mostly assist institutions in preparing for future adjustments in the definitions of surveillance [2]. For example, patients with two catheters in future might count for two days instead of one day. Ventilators have the capacity to transmit data electronically with accurate information concerning the exposures of patients. Surveillance systems with the capacity of accommodating device data in electronic form could enhance denominator computations' correctness in addition to its efficiency [14]. Lastly, the capacity to capture the days of patients by unit is essential when determining the rates of devicerelated rates [14].

\section{2) Numerator Data Capturing:}

Infection preventionists typically refer to records by the patients' bedsides and/or clinical officers to offer clinical settings data with conventional surveillance. Conversely, electronic systems are capable of making determinations using comparatively scarce data [4]. Despite access to the completely clinical chart and treatment of clinician expressions being alluring, these sources appear problematic. This is because the probability for inter-institutional variations in availability of data, distinctions in reviewer rigor in reviewing records, prejudices in accessing data to back a predefined outcome, and differences in data interpretation exists [2]. Moreover, consultation of clinicians can prove particularly deceitful since they often champion their often subjective clinical gestalt as opposed to evaluating whether patients satisfy the definition criteria for formal 
surveillance [15].

While considering the data components for inclusion in the electronic surveillance numerator, it is important to consider the extent to which data is available, accessible, and the impact such data has on reliability and accuracy [2]. The availability of data involves the consistency of electronic documentation and workflow processes across the institution. For instance, results of blood culturing are considerably available and their collection techniques are comparatively constant within different centers. Conversely, catheter-tip cultures depend on local practices considerably and therefore examples of low availability of data. Data accessibility speaks to the ease of access of the format data storage assumes [1]. For instance, majority of data feeds pertaining to microbiology include organisms as discrete elements. Examples of low data accessibility instances include records that are difficult to compute readily and depend on text processing for the extraction of preferred data components.

\section{3) Discrete Data Capturing:}

Woeltje et al. [2] alluded to the possibility of collecting patient information in EHRs from different locations and in numerous ways. To guarantee reliability and effectiveness, electronic algorithms must be fed with discrete data that has well-defined meanings. SilowCarroll, Edwards, and Rodin [16] indicated that existence of requisite data in discrete fields renders EHRs most useful. This is because data contained in discrete fields is easy to aggregate, sort, and manipulate. On the other hand, data exclusively existent in free-text fields needs manual intermediation when extracting and analyzing [16]. EHRs capture discrete data most readily by restraining the entry options through dropdown lists, checkboxes, or radio buttons [2].

During the design and implementation of EHRs, some hospitals have been deliberately structuring data fields to optimize their capabilities and sidestep text fields [16]. Other hospitals have been deferring to the preferences of their physicians for free-text data fields. This compels such hospitals to depend on quality review staff to go through notes made by clinicians to manually source for usable information [16]. Abdallah et al. [17] demonstrated that discrete data fields enhance usage of compliance reports and performance dashboards. Full automation of infection detection by using discrete data components captured through electronic medical records would render most reliability problems marring systems dependent on human interpretation obvious [18].

In other words, automated systems would not be subject to cognitive biases, deliberate manipulation of infection rates, and strategies of modelling would be applied in estimating event probabilities as opposed to no/yes determinations [18]. Probability estimations through clinical parameter modelling needs to avoid the rapidly raised infection rates that are observable through infection detection algorith mically [19]. Instead of binary algorithmic grouping, total rates would be produced through summing of estimated probabilities [20].
Despite the numerous benefits associated with enhanced EHR performance due to discrete data usage, the approach is not devoid of limitations. One of the main concerns associated with restrained data entry choices is that through restrained options, clinically important nuances may no longer be represented within the documentation. Altogether it is possible to overcome this limitation by enabling free text note entry fields to accompany the discrete data every time this makes sense clinically [16]. According to Woeltje et al. [2], tools for processing natural language that are capable of transforming sophisticated, free text to discrete data are underdoing constant improvement and appear promising. However, they are not available to non-expert users readily.

\section{B. Data Needs for Different ICU HAI Surveillance}

ICUs could benefit significantly from clinical data warehousing because they qualify as information-rich environments that have a high information technology and automation degree. Moreover, ICUs have certain traits that render clinical data warehousing even more relevant and interesting [13]. These characteristics include high dependency and access to data and technology, importance of access to and cost of intensive care to management matters, obligatory accountability for quality of care, and introduction of intensive care medicine as an autonomous discipline. Due to all these intensive care characteristics, the need to assess ICU data from different levels and diverse views is outspoken. Yet, the most common HAIs in the ICU have different denominator, numerator, and discrete data collection needs. In the subsequent sections below, focus shifts to four main ICU HAIs, including Central Line-Associated Bloodstream Infection (CLABSI), Urinary Tract Infection (UTI), Surgical Site Infections (SSIs) and Ventilator-Associated Conditions/Events (VACs/VAEs) respectively.

\section{1) Central Line-Associated Bloodstream Infection:}

The larger fraction of initial works concerning semiautomated and fully automated HAI surveillance uses CLABSI [21]. Denominator data capturing for CLABSI needs collecting updated CVC as discrete data for determining line-days. On the other hand, a positive result on blood culture is a prerequisite for numerator data required for CLABSI. This is now a comparatively easy incident for electronic capturing. Nonetheless, specific organism needs capturing and comparing against different cultures for determining the extent to which the respective organism implies skin contamination as opposed to presenting pathogen-like traits [22]. Currently, fully- and semi-auto mated electronic surveillance systems depend upon positive specimen cultures obtained from non-blood body sites to assist in assessing whether a positive culture of the blood denotes secondary infections [12].

It is possible to categorize some secondary Blood Stream Infections (BSIs) as CLABSIs. This is due to cultures obtained from potential principal locations like 
intraabdominal inflammations being unavailable or lacking affirmative results [2]. Additionally, patients with neutropenia and mucositis could present with translocation of microorganisms in the gut to the bloodstream resulting in inaccurate categorization of the bacteremias as CLABSIs [23]. In future, definitions of surveillance could consider these confounding clinical situations when evaluating the extent to which a culture implies a CLABSI [2].

Presently, determination of the presence of conditions as those described in the previous paragraph from discrete data could be considerably difficult. Increasingly reliable in formation could be availed electronically as the maintenance of comprehensive and accurate lists of patient problems becomes widely accepted as standard practice during documentation of care in EHRs. Subsequently, integration of such information into algorithms in future could enhance specificity. Semiautomated as well as fully-automated algorithms of electronic surveillance purposed for extensive usage need to evaluate the availability of requisite data entry components from EHR systems in hospitals [24].

\section{2) Urinary Tract Infections (UTIs):}

Catheter-Associated Urinary Tract Infection (CAUTI) was revised into asymptomatic and symptomatic infections in 2009 [25]. It is no longer possible to report asymptomatic infections that have no secondary bacteremia whereas the rates of infection appear in reports as symptomatic UTIs for every 1,000 device-days (catheter) [26]. To determine the denominator data, the process resembles the CVC device-days although electronic urinary device-days' capture needs explicit documenting within the electronic system [14]. To allow for semi- and fully-auto mated surveillance, device data at individual patient levels is crucial. This is attributable to the fact that the only infections measured are those from patients with urinary catheters for certain timeframes. In the CAUTI context, the capacity to collect patient-days as units is vital from the standpoint of measuring device utilization. Furthermore, it is also important when alternate denominators become standard [14].

For capturing nu merator data, the data components that describe the clinical symptoms of patients are important when applying NHSN definitions [2]. These data components include temperature, dysuria, temperature, frequency, costovertebral angle tenderness or pain, and suprapubic tenderness. There are requirements for extra data for children that are no more than a year old [25]. Temperature and age being comparatively easy points of data to capture structurally in general. However, the rest of the criteria are increasingly subjective as well as considerably problematic in terms of extraction and interpretation out of records kept electronically. Preferably, these elements of data need inclusion in nursing assessment and may be documented in the form of discrete data in the sheets used for assessment. Owing to the difficulties involved in the application of biased clinical data when defining a case, CAUTI assessments in future may move outside subjective components. Within all systems of surveillance, variation pertaining practice concerning collation of urine cultures could wield intense impacts on the rates reported [2].

Concerning discrete data, laboratory data elements are necessary for detecting CAUTIs algorithmically. Examples of laboratory data elements include urine cultures, microscopic pyuria, Gram stains, and laboratory outcomes concerning nitrate and leukocyte esterase [25]. Microscopic pyuria data involves urinary specimens that have at least $10 \mathrm{WBC} / \mathrm{mm} 3$ or at least $3 \mathrm{WBC} /$ highpower unspun urine field [25]. Currently, the NHSN criteria disallows cultures containing at least three organisms and recommend differential symptom evaluations based on whether the culture contains at least 103 or at least 105 colony-forming units/ml [25]. Consequently, the count of distinctive types garnered for every culture as well as the reported quantities are essential elements of data when identifying CAUTI algorithmically.

\section{3) Surgical Site Infections (SSIs):}

Unlike denominators applicable in device-related infections where aggregate device data is applied, SSIs denominators require gathering of specific data elements at the individual levels of patients for each individual undergoing surgical procedures to ensure appropriate risk adjustment [2]. Despite earlier risk adjustment using somewhat basic data like wound class, surgery duration, and the American Society of Anesthesiologists score, recent NHSN models of risk adjustment need more information about patients. The electronic submission of denominator data requires formatting and storing the information in such a manner that is compliant with extraction electronically [27]. It is imperative to be keen about data validity. Although surgical information systems are capable of capturing wound class, staff in the operating rooms may preferentially enter the most usual values as default values for every case. Documentation procedures and expectations need clear definitions in response to incidences taking place in course of the case. This may alter the class of the wound while making sure that capturing of data happens [2].

Owing to the complex nature of defining SSIs, full automation of electronic surveillance for capturing numerator data can be challenging. Altogether, semiautomated electronic surveillance could be helpful when determining cases, which ends up reducing the whole surveillance task. Nonetheless, combining codes for SSIs (i.e. administrative data) in addition to antibiotic and readmission data are famous for improving SSI case finding [28]. Despite the fact that the result of a positive culture is not necessary for SSI diagnosis, the presence of a wound culture in postoperative patients could be an indication for finding cases. Similarly, readmission in 30 days after an ordinary surgical procedure or 90 days following a surgical procedure involving an implant could signal potential infection [29].

Woeltje et al. [2] argued that employing different algorithms for various surgical procedures often proves helpful in optimizing specificity and sensitivity. Indeed, 
Koek et al. [29] confirmed that SSIs' identification is multidimensional. Various authors including Tanner et al. [30]; Calderwood et al. [31] concur that focus on inpatient data exclusively from the original surgical admission is inadequate. This is despite inpatient data being homogenous across several hospitals. With hospital stays becoming progressively shorter, an increasing fraction of SSIs becomes noticeable post-discharge. Thus, post-discharge surveillance is now inevitable for determining SSI incidence [29]. Without undertaking post-discharge surveillance, the potential for significant underestimation of SSIs' incidence is quite high [31]. Moreover, hospital comparisons could be faulty [29].

\section{4) Ventilator-Associated Conditions /Events (VACs/VAEs):}

At the beginning of 2013, NHSN unveiled novel characterizations regarding Ventilator-Associated Conditions designed to overcome most weaknesses characterizing conventional ventilator-associated pneumonia definitions involving their inaccuracy, complexity, and subjectivity [32]. These new definitions shifted the surveillance focus from pneumonia exclusively to mechanical ventilation complications generally [32]. The altercation is an increasingly precise depiction of diagnostic demerits associated with repeated surveillance. This constitutes a chance for broadening concentration of prevention away from pneumonic conditions exclusively to including multiple critical care problems that are compliant with automation. It is noteworthy that the new definitions exclude radiographic criteria based on their difficulty to interpret, susceptibility to extensive observer disagreements and difficulty in parsing electronically [2].

The new VAE (Ventilator-Associated Event) surveillance protocol has three tiers. The first tier entails determination of VAC based on respiratory worsening based on Positive Expiratory End Pressure (PEEP) or $\mathrm{FiO} 2$. Upon meeting $\mathrm{VAC}$, the second tier is evaluated for incidence of IVAC (Infection-Related VentilatorAssociated Complication) using inflammatory signs [22]. When IVAC is found, the third tier using microbiological data is evaluated for a probable Ventilator-Associated Pneumonia (VAP). Woeltje et al. [2] described the new surveillance paradig $\mathrm{m}$ as anchoring on IVAC and VAC. Moreover, they are the only measures proposed by CDC as important considerations for public reporting. Altogether, CDC experts allude to possible VAP usage for purposes of enhancing internal quality. The new VAE algorith $m$ has specific criteria that appears promising for extraction of electronic data and data mining for identification of cases [22].

Denominator data for VAE surveillance, which entails patient days and ventilator days, can be garnered from patients. The inpatient locales that qualify for participation in VAE surveillance are adult locations within inpatient facilities for rehabilitation, long-term acute care hospitals, and acute care hospitals [22]. The locations include ICUs, SCAs (Specialty Care Areas), long-term care units, wards, and step-down units. Notably, monitoring of VAEs post discharge is necessary when patients transfer to other facilities while on mechanical ventilation still. Altogether, VAEs found within 2 days after discharge with day 1 being day of discharge need to be reported to NHSN without any additional ventilator days being reported [22].

Numerator data for VAEs has three main constituents. These include inpatient demographics, mechanical ventilation start date, and location where mechanical ventilation commenced [22]. In addition, numerator data may include information concerning patient death if applicable, antimicrobial vulnerabilities, organis ms detected, and whether patients developed secondary infections in the bloodstream [22]. The computation of the rate of VAE per 1000 ventilator days results through division of the VAEs' count by the ventilator days and multiplying the result by 100 , which denotes the mechanical ventilation episodes [22].

\section{GUIDELINESFOR DIFFERENT ICU HAI SURVEILLANCE}

Following the review of the data requirements for the surveillance of the four main ICU HAIs, this section contains the guidelines for the surveillance of the same ICU HAIs under respective subsections.

\section{A. Central Line-Associated Bloodstream Infection}

In the case of CLABSIs, it is important to ensure structured documentation of all CVCs present including anatomic site and catheter type in the EHR. The approach may involve documenting the CVC presence daily or removal and insertion dates could be useful. It is noteworthy that the method chosen depends on local documentation and workflow practices. At the very least, vulnerability testing and positive microbiology culture need to be available and accessible as discrete data elements. Positive microbiology culture outcomes include identification of species and specimen site to the magnitude undertaken by the lab.

Due to the changes in definitions with time, additional information may be necessary to ensure fully automated surveillance electronically. Examples of changes include the recent addition of conditions for a mucosal barrier injury laboratory-BSI. The definition of the mucosal barrier injury requires evaluation. The evaluation entails determination of the extent to which an individual patient got a transplant of the allogeneic stem cell in the past 12 months, his/her count of white blood cells, as well as graft existence and seriousness compared to the host ailment. It would prove challenging for most systems when it comes to capturing requisite data electronically in order to ensure full auto mation with the determination of the injury of the mucosal barrier.

\section{B. Urinary Tract Infection (UTI)}

In developing an electronic surveillance system for CAUTI that is semi-automated, ensuring discrete capture of urinalysis data, quantitative microbiology data, and urinary catheter data at the bare minimum is important. Extra work is necessary for defining electronic 
surveillance algorith ms with semi-automation or even for full automation with enhanced accuracy. For as long as the subjective symptoms remain part of the definition, they need capturing in a structured way whenever this is possible.

\section{Surgical Site Infections (SSIs)}

Surgical information systems and/or EHRs should be capable of identifying all patients receiving target surgeries. They also need to be capable of capturing risk adjustment specific to a patient such as surgery duration, basal metabolic index, antibiotic administration, selected comorbidities, and wound class. Billing codes such as CPT, ICD-10 and ICD-9 may be applied in the classification of surgical procedures into categories of NHSN. Information concerning wound culture, readmission, admin istrative codes, and antibiotic administration are more recommended for capturing numerator data. On the other hand, access to claims or EHR data from offices of physician outpatients would be useful during post-discharge surveillance. Altogether, there is need for further work in defining algorithms with better sensitivity for electronic surveillance with the capacity to optimize tasks pertaining to finding cases and simultaneously decreasing the load of surveillance.

\section{Ventilator-Associated Conditions/Events (VACs/VAEs)}

The revised VACs/VAEs definitions are amenable to both fully automated and semi-automated surveillance systems. To achieve this, it is mandatory that several sets of data be captured. These data sets include the daily minimu $\mathrm{m}$ PEEP and $\mathrm{FiO} 2$, daily maximu $\mathrm{m}$ and minimu $\mathrm{m}$ WBC count and temperature, and days when antibiotics were started and when they were stopped. For the application of the probable and possible definitions of VAP, counts of epithelial cells and Gram stain neutrophil (either semi-quantitative or absolute) and either semiquantitative or quantitative results of pulmonary culture are necessary. The monthly device-days (ventilator) may come from the data stream in the form of total day-to-day values of PEEP/unit in a month.

\section{DISCUSSION AND CONCLUSIONS}

Despite still being at infancy, electronic information control surveillance systems for HAIs are improving and becoming more commonplace as the acceptance levels rise. One of the most outstanding motivators informing the transition from conventional manual information control surveillance to fully automated ones is the susceptibility of tradition methods to variability that is attributable to variations between hospitals and infection preventionists in their surveillance intensity. Additionally, traditional surveillance systems are subject to personal interpretation biases. Both bias and variability compromise reliability. By extension, poor reliability weakens the degree of validity in inter-institutional comparis ons [2].

Despite the fact that episodic audits, rigorous training, and retention are, in theory, capable of achieving acceptable reliability, the manpower necessary for realizing this performance level across various devicerelated infections in a broad array of diverse types of ICUs across many hospitals is almost improbable to conceive. Concerning the data requirements, numerator data capturing as achievable through bedside clinician records and devices is subject to the reliability of the data entry. On the other hand, denominator data is dependent upon tallying of patient-days and device days in general. Whereas this is an increasingly common task with high probability for accuracy, the validity of at-risk patients, procedures, and days is the basis for reliable denominator data. Finally, discrete data is likely to enhance the performance of electron ic surveillance of HAIs in ICUs. Avoiding free text data renders EHRs more reliable although it is difficult at times to enter constrained data for some conditions and this could account for why some hospitals deter this role to their physicians.

In relation to electronic surveillance data requirements, specific to different, common ICU-related HAIs, this review pointed to different requirements for each of the HAIs. For CLABSIs, it was established that denominator data involves garnering of current CVC as discrete data for the purposes of determining line-days while positive blood cultures are a prerequisite for numerator data. Although capturing discrete data concerning CLABSIs is a challenge, neutropenia and mucositis conditions as detected in patients could prove useful especially where blood cultures could render BSI classifying as BSIs.

For CAUTIs, denominator data resembles CVC catheter-days although electronic urinary catheter-days' at patient level. Numerator data should describe the clinical symptoms of patients including temperature, dysuria, temperature, frequency, costovertebral angle tenderness or pain, and suprapubic tenderness. However, CAUTI numerator data for children below a year have additional require ments. Discrete data includes laboratory data elements including urine cultures, microscopic pyuria, Gram stains, and leukocyte esterase and nitrate. For the guidelines, discrete capture of urinalysis data, quantitative microbiology data, and urinary catheter data at the bare minimum developing a semi-automated CAUTI electronic surveillance system.

SIs denominators require gathering of specific data elements at the individual levels of patients with recent NHSN models of risk adjustment needing more patient information. The complex nature of defining SSIs makes capturing numerator data can be challenging. However, combining codes for SSIs including antibiotic and readmission data are famous for improving SSI case finding. Focusing on inpatient data exclusively from the original surgical admission is inadequate. The data guidelines for surgical in formation systems and/or EHRs include capability to capture all patients receiving target surgeries, risk adjustment specific to patients like time taken in surgery, basal metabolic index, antibiotic administration, carefully chosen comorbidities, and wound class. Altogether, wound culture, readmission, administrative codes, and antibiotic administration are more preferable for capturing numerator data. 
Denominator data for VAE surveillance entails patient days and ventilator days. Numerator data for VAEs has three main constituents including inpatient demographics, mechanical ventilation start date, and location where mechanical ventilation commenced. Additional data includes patient death, antimicrobial vulnerabilities, organisms detected, and development of secondary infections in the bloodstream. VACs/VAEs guidelines involve mandatory capturing of the daily minimu $\mathrm{m}$ PEEP and $\mathrm{FiO} 2$, daily maximu m and minimum WBC count and temperature, and commencement and termination days of antibiotics. Epithelial cells, Gram stain neutrophil and semi-quantitative or fully quantitative results of pulmonary culture are necessary. Device-days (ventilator) are obtainable from the stream of data in the format of total of day-to-day values of PEEP/unit in a month.

The shift to objective definitions and the development of electronic systems for automating analysis, extraction, and presentation of data will render surveillance more effective. Yet, it also promises the ability to direct most of the already limited resources towards prevention as opposed to exclusive counting of infections. In conclusion, stakeholders in infection control and prevention need to adopt and advocate for unbiased electronic definitions that are amenable to/with fully automated surveillance.

\section{REFERENCES}

[1] A. Palumbo, P. Loveless, M. Moll and S. Ostroff, 'Evaluation of Healthcare-Associated Infection Surveillance in Pennsylvania Hospitals', Infect Control Hosp Epidemiol, vol. 33, no. 02, pp. 105-111, 2012.

[2] K. Woeltje, M. Lin, M. Klompas, M. Wright, G. Zuccotti and W. Trick, 'Data Requirements for Electronic Surveillance of Healthcare-Associated Infections', Infect Control Hosp Epidemiol, vol. 35, no. 09, pp. 1083-1091, 2014.

[3] T. Lee, O. Montgomery, J. Marx, R. Olmsted and W. Scheckler, 'Recommended practices for surveillance: Association for Professionals in Infection Control and Epidemiology (APIC), Inc.', American Journal of Infection Control, vol. 35, no. 7, pp. 427-440, 2007.

[4] R. Freeman, L. Moore, L. García Álvarez, A. Charlett and A. Holmes, 'Advances in electronic surveillance for healthcare-associated infections in the 21st Century: a systematic review', Journal of Hospital Infection, vol. 84, no. 2, pp. 106-119, 2013.

[5] J. Doherty, L. Noirot, J. May field, S. Ramiah, C. Huang, W. Dunagan and T. Bailey, 'Implementing GermWatcher ${ }^{\mathrm{TM}}$, an Enterprise Infection Control Application', American Medical Informatics Association (AMAI) Annual Symposium Proceedings, pp. 209-213, 2006.

[6] K. Nelson and C. Williams, Infectious disease epidemiology. Sudbury, Mass.: Jones and Bartlett Publishers, 2007.

[7] Manetho. and W. Waddell, Manetho. Cambridge, Mass.: Harvard University Press, 1940. Retrieved from https://ia800307.us.archive.org/26/items/manethowithengli 00maneuoft/manethowithengli00maneuoft.pdf

[8] B. Choi, 'The Past, Present, and Future of Public Health Surveillance', Scientifica, vol. 2012, pp. 1-26, 2012.

[9] R. Merrill, Introduction to epidemiology, 4th ed. Jones \&
Bartlett Publishers, 2010.

[10] R. Pearl, Introduction to medical biometry and statistics. Philadelphia: W.B. Saunders, 1923.

[11] A. Boonstra and M. Broekhuis, 'Barriers to the acceptance of electronic medical records by physicians from systematic review to taxonomy and interventions', BMC Health Services Research, vol. 10, no. 1, p. 231, 2010.

[12] K. Woeltje, K. McMullen, A. Butler, A. Goris and J. Doherty, 'Electronic Surveillance for HealthcareAssociated Central Line-Associated Bloodstream Infections Outside the Intensive Care Unit', Infect Control Hosp Epidemiol, vol. 32, no. 11, pp. 1086-1090, 2011.

[13] M. de Mul, P. Alons, P. van der Velde, I. Konings, J. Bakker and J. Hazelzet, 'Development of a clinical data warehouse from an intensive care clinical information system', Computer Methods and Programs in Biomedicine, vol. 105, no. 1, pp. 22-30, 2012.

[14] M. Wright, A. Fisher, M. John, K. Reynolds, L. Peterson and A. Robicsek, 'The electronic medical record as a tool for infection surveillance: Successful automation of device-days', American Journal of Infection Control, vol. 37, no. 5, pp. 364-370, 2009.

[15] L. Backman, G. Nobert, R. Melchreit, R. Fekieta and L. Dembry, 'Validation of the surveillance and reporting of central line-associated bloodstream infection denominator data', American Journal of Infection Control, vol. 42, no. 1, pp. 28-33, 2014.

[16] S. Silow-Carroll, J. N. Edwards and D. Rodin, 'Using electronic health records to improve quality and efficiency: The experiences of leading hospitals.', The Commonwealth Fund, vol. 17, pp. 1-39, 2012.

[17] S. Abdallah, M. Mahmoud, N. El-Tayeb and M. Abdel Magid, 'Designing and Implementing of Electronic Health Record System in Ksa using Sq1 \& Asp.Net', Innovative Systems Design and Engineering, vol. 6, no. 1, 2015.

[18] W. Trick, 'Decision Making During Healthcare-Associated Infection Surveillance: A Rationale for Automation', Clinical Infectious Diseases, vol. 57, no. 3, pp. 434-440, 2013.

[19] W. Trick, B. Zagorski, J. Tokars, M. Vernon, S. Welbel, M Wisniewski, C. Richards and R. Weinstein, 'Computer Algorithms To Detect Bloodstream Infections', Emerg. Infect. Dis., vol. 10, no. 9, pp. 1612-1620, 2004.

[20] M. van Mourik, K. Moons, W. van Solinge, J. Berkelbachvan der Sprenkel, L. Regli, A. Troelstra and M. Bonten, 'Automated Detection of Healthcare Associated Infections: External Validation and Updating of a Model for Surveillance of Drain-Related Meningitis', PLoS ONE, vol. 7, no. 12, p.e 51509, 2012.

[21] B. Hota, M. Lin, J. Doherty, T. Borlawsky, K. Woeltje, K. Stevenson, Y. Khan, J. Young, R. Weinstein and W. Trick, 'Formulation of a model for automating infection surveillance: algorithmic detection of central-line associated bloodstream infection', Journal of the American Medical Informatics Association, vol. 17, no. 1, pp. 42-48, 2010.

[22] T. Horan, M. Andrus and M. Dudeck, 'CDC/NHSN surveillance definition of health care-associated infection and criteria for specific types of infections in the acute care setting', American Journal of Infection Control, vol. 36, no. 5, pp. 309-332, 2008.

[23] J. Lukenbill, L. Rybicki, M. Sekeres, M. Zaman, A. Copelan, H. Haddad, T. Fraser, M. DiGiorgio, R. Hanna, H. Duong, B. Hill, M. Kalaycio, R. Sobecks, B. Bolwell and E. Copelan, 'Defining Incidence, Risk Factors, and Impact on Survival of Central Line-Associated Blood Stream Infections Following Hematopoietic Cell 
Transplantation in Acute Myeloid Leukemia and Myelodysplastic Syndrome', Biology of Blood and Marrow Transplantation, vol. 19, no. 5, pp. 720-724, 2013. K. Cato, B. Cohen and E. Larson, 'Data elements and validation methods used for electronic surveillance of health care-associated infections: A systematic review', American Journal of Infection Control, vol. 43, no. 6, pp. 600-605, 2015.

[24] National Healthcare Safety Network (NHSN). NHSN manual for CAUTI surveillance. Retrieved from: http://www.cdc.gov/nhsn/PDFs/pscM anual/7pscCAUTIcur rent.pdf

[25] National Healthcare Safety Network. National Healthcare Safety Network newsletter, 6(1) ,2011. Retrieved from: http://www.cdc.gov/nhsn/PDFs/Newsletters/NHSN_NL_ MAR_2011_final.pdf

[26] H. Hsu, E. Shenoy, D. Kelbaugh, W. Ware, H. Lee, P. Zakroysky, D. Hooper and R. Walensky, 'An electronic surveillance tool for catheter-associated urinary tract infection in intensive care units', American Journal of Infection Control, vol. 43, no. 6, pp. 592-599, 2015.

[27] D. Yokoe, Y. Khan, M. Olsen, D. Hooper, M. Greenbaum, J. Vostok, J. Lankiewicz, V. Fraser and K. Stevenson, 'Enhanced Surgical Site Infection Surveillance Following Hysterectomy, Vascular, and Colorectal Surgery', Infect Control Hosp Epidemiol, vol. 33, no. 08, pp. 768-773, 2012.

[28] M. Koek, J. Wille, M. Isken, A. Voss and B. van Benthem, 'Post-discharge surveillance (PDS) for surgical site infections: a good method is more important than a long duration', Eurosurveillance, vol. 20, no. 8, p.21042, 2015.

[29] J. Tanner, W. Padley, M. Kiernan, D. Leaper, P. Norrie and R. Baggott, 'A benchmark too far: findings from a national survey of surgical site infection surveillance', Journal of Hospital Infection, vol. 83, no. 2, pp. 87-91, 2013.

[30] M. Calderwood, K. Kleinman, D. Bratzler, A. Ma, C. Bruce, R. Kaganov, C. Canning, R. Piatt and S. Huang, 'Use of Medicare Claims to Identify US Hospitals with a High Rate of Surgical Site Infection after Hip Arthroplasty', Infect Control Hosp Epidemiol, vol. 34, no. 01, pp. 31-39, 2013.

[31] S. Magill, M. Klompas, R. Balk, S. Burns, C. Deutschman, D. Diekema, S. Fridkin, L. Greene, A. Guh, D. Gutterman, B. Hammer, D. Henderson, D. Hess, N. Hill, T. Horan, M. Kollef, M. Levy, E. Septimus, C. VanAntwerpen, D. Wright and P. Lipsett, 'Developing a new national approach to surveillance for ventilator-associated events: Executive summary', American Journal of Infection Control, vol. 41, no. 11, pp. 1096-1099, 2013.

\section{Authors' Profiles}

Manal Abumelha received her bachelor degree in 2011 from King Khalid University, KSA. Since November 2011, she has been working as a Teaching Assistant in King Khalid niversity. Now she is conducting to finish her Master degree in King Abdulaziz University. Her research interest includes enterprise architecture.

Awatef Hashbal Zarra got her bachelor degree in 2010 from King Khalid University, Kingdom of Saudi Arabia. She worked as a Teaching Assistant since 2011 in King Khalid University. She was quality coordinator of Information Sy stem Department for two years. She has a great interest in the field of big data analytics as well as e-learning and she created King Khalid University in Second Life (SL) virtual environment in 2010. During 2016, she will finish her Master degree from King Abdulaziz University.

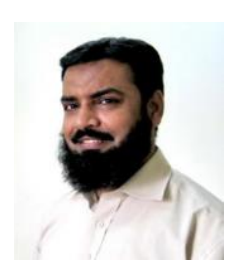

Farrukh Nadeem received his Ph.D. in 2009 from the University of Innsbruck, Austria. Since July 2009, he has been working as an Assistant Professor. His main research interests include performance modeling and prediction, and scheduling scientific workflows in distributed systems, particularly the Grid and the Cloud. He has been involved in a several Austrian and Saudi research and development projects. Farrukh is author of more than 24 papers, including four book chapters. He holds several distinctions and awards during his educational career.

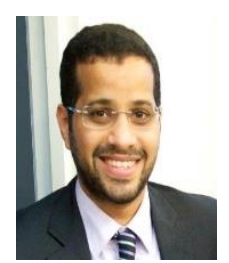

Naif Aljohani is Assistant Professor at the Faculty of Computing and Information Technology in King Abdul Aziz University, Jeddah, Saudi Arabia. He holds a PhD in Computer Science from the University of Southampton, UK. He received the Bachelor's degree in Computer Education from King Abdul Aziz University, 2005. In 2009, he received the Master degree in Computer Networks from La Trobe University, Australia. His research interests are in the areas of mobile and ubiquitous computing, mobile and ubiquitous learning, learning and knowledge analytic, semantic web, Web Science ,technology enhanced learning and human computer interaction.

How to cite this paper: Manal Abumelha, Awatef Hashbal, Farrukh Nadeem, Naif Aljohani, "Development of Infection Control Surveillance System for Intensive Care Unit: Data Requirements and Guidelines", International Journal of Intelligent Systems and Applications (IJISA), Vol.8, No.6, pp.19-26, 2016. DOI: $10.5815 /$ ijisa.2016.06.03 